\title{
Extraoral approach for removing the intraparotid large sialolith. A case series reports
}

\author{
Artur Papikyn ${ }^{1,4}$, Hayk Papikyan ${ }^{2}$, Nahapet Eghiazaryan ${ }^{3}$ and Hakobyan Gagik ${ }^{4 *}$ \\ ${ }^{1}$ Department of Oral and Maxillofacial Surgery, Yerevan State Medical University after M. Heratsi, Armenia \\ ${ }^{2}$ Department of Oral and Maxillofacial Surgery, Mikayelyan University Hospital, Yerevan, Armenia \\ ${ }^{3}$ Yerevan State Medical University after M. Heratsi, Armenia \\ ${ }^{4}$ Department of Oral and Maxillofacial Surgery Yerevan State Medical University after M. Heratsi, Armenia
}

\begin{abstract}
Salivary duct lithiasis refers to the formation of calcareous concretions or sialoliths in the salivary duct causing obstruction of salivary flow resulting in salivary ectasia, sometimes even dilatation of the salivary gland. They are most common in the submandibular gland and they less frequently developed in parotid gland. Sialoliths affecting the parotid glands are usually small, unilateral and can be symptomatic or asymptomatic and these are highly radiolucent.

In this case report, the treatment of a 65 -year-old patient with parotid sialolithiasis atypical location was presented. Computed tomography showed a lesion in left cheek area, and tiny calcification was noted within internal low-density portion of cheek mass. These findings suggested sialolithiasis measuring $5,6 \mathrm{~mm}$ in left parotid gland with duct dilatation. That was surgically removed by extraoral access after determining its correct location by using computerized tomography imaging. After surgery patients expressed satisfaction with the result of treatment and improved quality of life. Extraoral approach for removing the intraparotid large sialolith has been demonstrated to be a reliable technique with good long-term results.
\end{abstract}

\section{Introduction}

Salivary duct lithiasis refers to the formation of calcareous concretions or sialoliths in the salivary duct causing obstruction of salivary flow resulting in salivary ectasia, sometimes even dilatation of the salivary gland. This also may be complicated by infection of the salivary gland which may result in chronic sialadenitis [1].

Parotid gland stone incidence in males to females is 2:1. It generally occurs at 3 rd to 6 th decades of life. Intraductal sialolith have more incidence of occurrence than intraglandular sialoliths [2]. Parotid calculi are unilateral, generally seen in duct and size is less than $1 \mathrm{~cm}$. Sialolith which are not detected by radiograph may require sialoendoscopy as $40 \%$ of parotid and $20 \%$ of submandibular stones are not radioopaque. More than $80 \%$ of salivary sialoliths occur in the submandibular duct or gland, $6-15 \%$ occur in the parotid gland and around $2 \%$ are in the sublingual and minor salivary glands [3]. The exact etiology and pathogenesis of salivary calculi is not known! but it is thought that the more alkaline, viscous, mucus-rich saliva, which contains a higher percentage of calcium phosphates, in addition to the long and sinuous position of Wharton's duct, contributes to stasis making the submandibular salivary system more prone to the development of sialoliths than the parotid gland [4].

It is known that systemic diseases (gout, Sjögrens), medications (anticholinergics, antisialogogues), local trauma, head and neck radiotherapy [5], being elderly [6] and renal impairment [7] also can predispose patients to sialolith formation. It is estimated that sialolithiasis affects 12 of every 1000 patients in the adult population [8].

Salivary calculi grow by deposition at an estimated rate of 1-1.5 mm/year [9]. Sialoliths are most the common cause of acute and chronic infections of salivary glands. The resulting salivary stasis from stone formation allows bacterial ascent into the gland and then increases the risk of bacterial colonization and acute salivary gland infection. Because stones are more common in Wharton's duct, so are acute bacterial infections of the submandibular gland versus the parotid [10]. The aim of this study is to evaluate treatment success patients with parotid gland sialolithiasis and treated with extraoral surgical approach for removing the sialoliths.

\section{Materials and methods}

A total of 8 patients were admitted in the Maxillofacial Department with an ailment of swelling, pain and inflammation on one side restricted to the lower jaw region between 2015-2019. The age of patients at the time of treatment ranged from 43 to 65 years. All patients underwent a thorough clinical examination according to a generally accepted scheme. The location of the lesions in left cheek area (3 cases), right cheek area (5 cases). Preoperative radiographs including cone beam were obtained for initial screening and evaluation. A computerized tomography revealed sialoliths measuring $4.2-7.4 \mathrm{~mm}$. All patients underwent surgical treatment with removing the intraparotid sialolith.

${ }^{*}$ Correspondence to: Gagik Hakobyan, Department of Oral and Maxillofacial Surgery Yerevan State Medical University after M. Heratsi, Armenia, Tel: (+37410)271146; E-mail: prom_hg@yahoo.com

Key words: salivary gland calculi, parotid gland, parotitis, sialolithiasis, salivary stone

Received: January 21, 2020; Accepted: February 06, 2020; Published: February 10,2020 
All patients signed an informed consent for surgery and participation in scientific studies.

\section{Results}

No intra-operative or immediate post-operative complications were noted. The postoperative evolution of the patients was favorable. After 3 year of observation, clinical and radiological indices were stable. Complications of sialolithiasis including presence of secondary infections, abscess formation, stenosed saliva ducts, chronic sclerosing sialadenitis did not reveal. After surgery patients expressed satisfaction with the result of treatment and improved quality of life.

This case report presents extraoral approach for removing the intraparotid large sialolith.

\section{Case report}

A 65-year-old patient came to the department of Oral \& Maxillofacial Surgery with complaint of pain, dryness in the mouth and swelling in the left parotid area that gradually increases during mastication. Patient noticed it 2 months back. The pain was localized, pricking in nature continuous and aggravated at mealtimes. There was no history of trauma. The patient has type 2 diabetes, hypertension, and uses antiplatelet agents. On extraoral examination, the patient had facial asymmetry due to a slight swelling on the left side of the face. The swelling was diffuse, The skin over the swelling was smooth, stretched. There were no secondary changes. Upon, physical examination, the left parotid region was diffusely swollen and painful to palpation. There was no cervical or other palpable lymphadenopathy. A purulent discharge was expelled from the left Stensen duct ostium during massaging of the gland and the ejection of saliva while milking the parotid gland was not as free flowing as it was on the other side. For a better assessment of diagnostic hypothesis, it was performed a computerized tomography of left parotid gland and revealed Sialolithiasis measuring $5.56 \mathrm{~mm}$ (Figures 1 and 2).

\section{Surgical technique}

The patient was hospitalized and under general endotracheal anesthesia it was performed an incision near the crease of the ear as in facelift and continued behind the ear (a lazy $S$ incision or modified Blair incision). A flap is made on the surface of the parotid gland to help expose the gland, the nerves and vessels were identified and saved. Then the stone was identified in the anterior part of gland 'in the main duct start point and was successfully removed (Figures 3-5). Closure was done with absorbable suture vicril 5.0 and with tight bandage to avoid leak of saliva. Patient took intravenous cephalosporin $1 \mathrm{~g}$ for 5 days, anti-inflammatory drugs. The patient was discharged after 7 days of hospital stay, free of symptoms and in good general conditions. Good clinical appearance after 1-month removal of intraparotid (Figure 6).
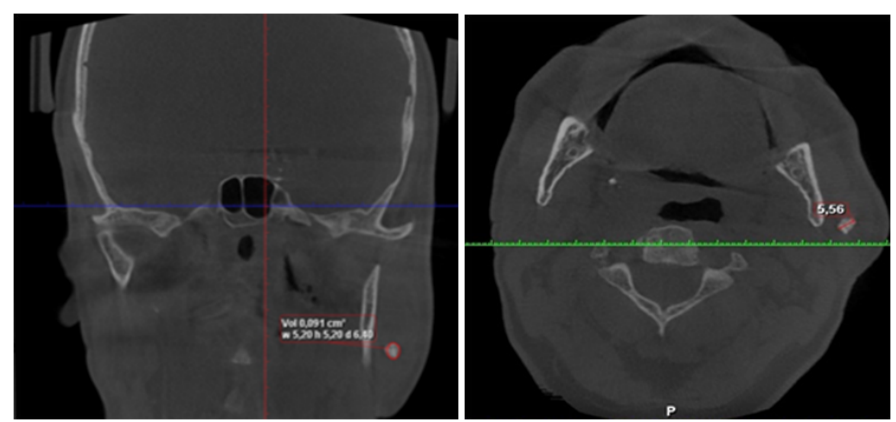

Figure 1. Preoperative CT scan showing the sialolith evident in the left parotid region

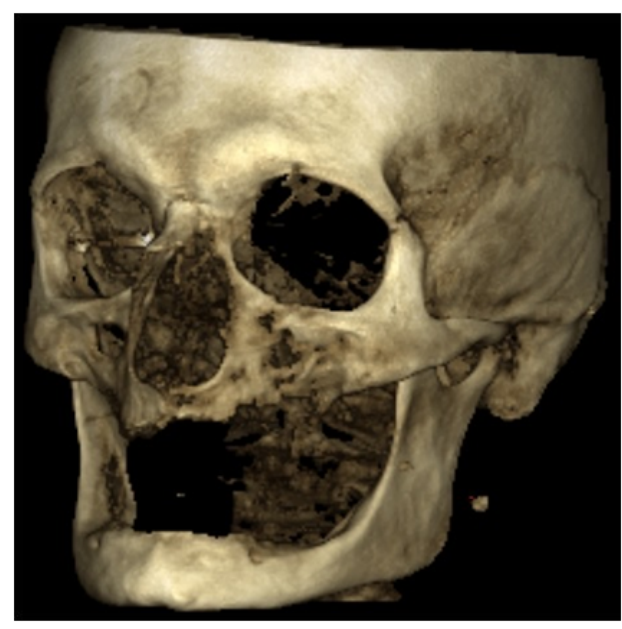

Figure 2. Three-dimensional reconstruction of the lesion site.

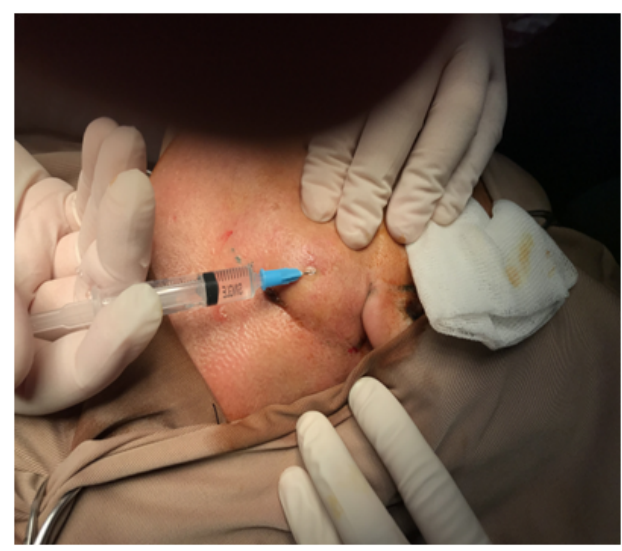

Figure 3. Hydroseparation with sol. Novocaine $0.5 \%-10 \mathrm{ml}$
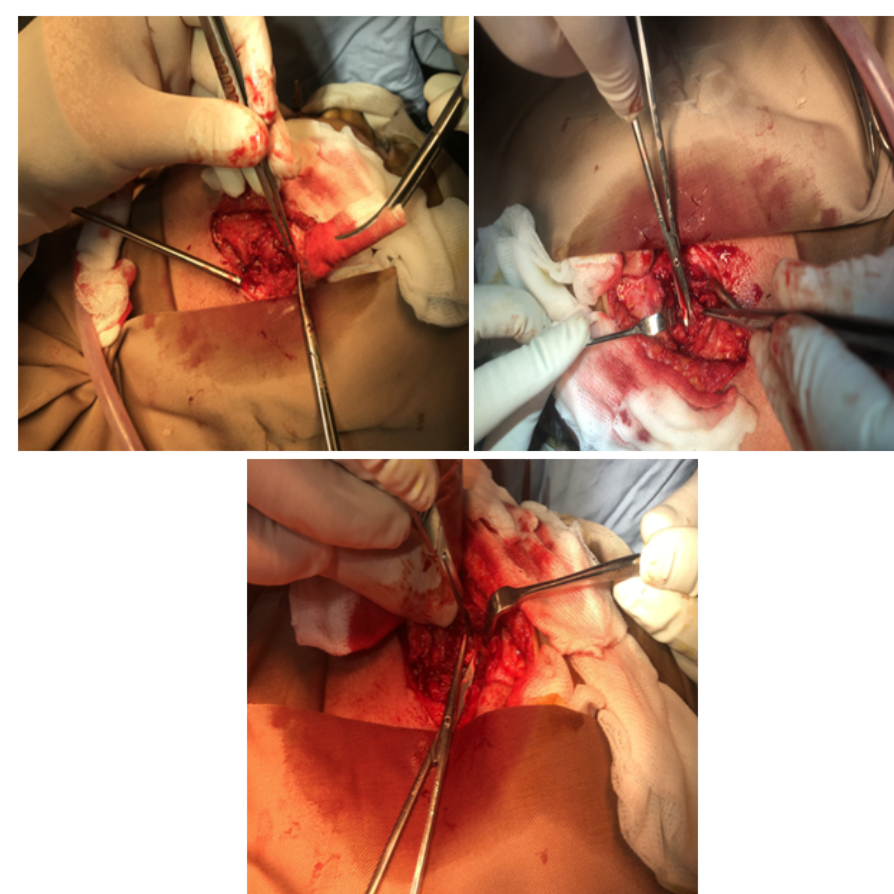

Figure 4. The sialolith was surgically removed under general anesthesia. Intraoperative view of the excision of the of the sialolith in the left parotid region via the standard extraoral approach, without any complications 


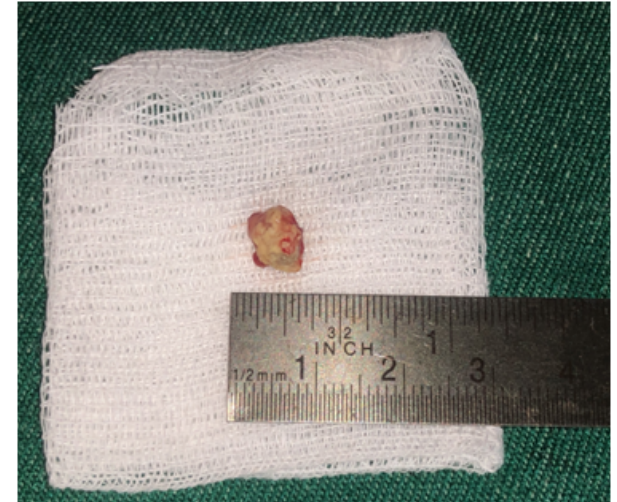

Figure 5. Large sialolith measuring $5.6 \mathrm{~mm}$ after surgically removed.

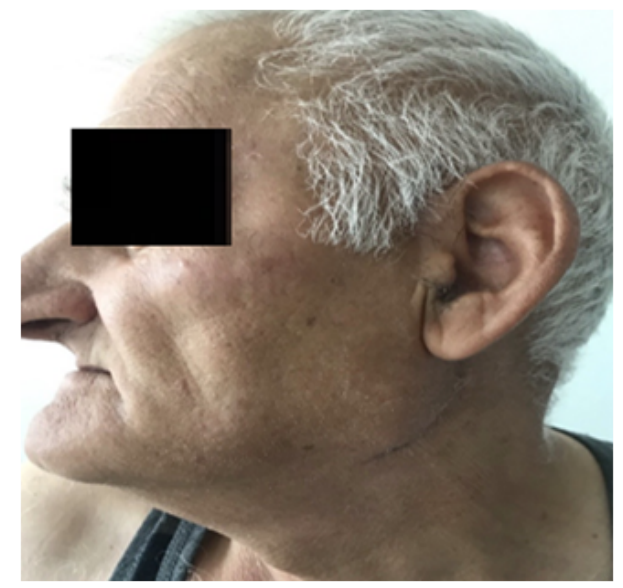

Figure 6. Clinical appearance after 1-month removal of intraparotid.

\section{Discussion}

Sialolithiasis is a relatively common disease of salivary glands, reported to account for up to $30 \%$ of this disease category, but is rarely observed in childhood and adolescence [11,12]. It was found that only $6.1 \%$ of 635 patients with sialolithiasis were younger than 20 years. Several theories have been suggested as to the pathogenesis of sialolithiasis, but none have yet been firmly established. The sialolith is assumed to be formed by the deposition of organic and inorganic materials around a central core. The central core is composed of the precipitation of calcium salts around an initial organic nidus that consists of altered salivary mucins, bacteria and desquamated epithelial cells. The organic layers are composed of various carbohydrates and amino acids and the inorganic layers consist of calcium phosphate, a small quantity of carbonates in the form of hydroxyapatite, and a small amount of magnesium and ammonia [13].

The ratio of organic to inorganic material in a submandibular stone is $18: 82$, whereas that in a parotid stone is $51: 49$ [14]. A combination of various factors is speculated to be involved in the formation of calculi. Chronic infection of the parotid gland and secondary trauma to its duct underlies the origin of calculus, whereas the anatomical and chemical properties of the submandibular gland predispose it to calculus formation, which further induces stagnation of saliva and invasion of bacteria [15].

One study proposed the role of foods, bacteria or foreign bodies within the oral cavity in stone formation. These may ascend the duct system and become the nidus for calcification combined with attracted inflammatory cells in the formation of parotid stones, whereas, mucus is thought to be the nidus in the case of submandibular stones [13].

Another study proposed that certain unknown metabolic abnormalities can increase the alkalinity of saliva, consequently promoting the crystallization of calcium and phosphate [16]. Stagnation of saliva, or physical trauma to the salivary gland or its duct may also play a role in calculus formation [17].

Systemic diseases have not been proved to be associated with sialolithiasis except for gout, although its calculi consist mainly of uric acid [13]. Nephrolithiasis was reported to be linked with sialolithiasis in up to $10 \%$ of patients in one study [11]. The predominance of submandibular stones can be explained by the following aspects. Anatomically, Wharton's duct is longer and wider than Stensen's duct, salivary flow is slow and against gravity, and chemically saliva is more alkaline and richer in calcium, phosphate and mucin proteins [17].

Careful attention should be paid to patient history and examination in order to avoid misdiagnosis. The differential diagnosis of parotid swelling or the radiopacities on radiographic examinations, could include viral sialadenitis (mumps), acute bacterial sialadenitis, lymphadenitis, dental abscess, human immunodeficiency virus sialopathy, Sjögren's syndrome, calcified lymph nodes, mainly after tuberculosis infection, phlebolithiasis, myositis ossificans and, finally, salivary gland tumors or metastasis [18]. Bimanual intraoral palpation is useful in detecting stones. In the direction of posterior to anterior, submandibular stones are palpable in the floor of the mouth, and parotid stones can be revealed around the orifice of Stensen's duct or along its course. Minor salivary stones are usually found in the buccal mucosa or upper lip [11].

Conventional and advanced imaging techniques have been developed in diagnosing sialolithiasis. Conventional intraoral X-ray may be useful but has limited application, since $40 \%$ of parotid and $20 \%$ of submandibular stones are radiolucent [14]. For parotid stones, the masseter muscle and the buccinator are additional obstacles to visualizing the calculi in Stensen's duct. In such cases, sialography is an adequate replacement modality which makes it possible to visualize the whole duct system. However, sialography is not viable in the setting of acute infection or in patients allergic to contrast medium. Nor it is indicated when the stone is located in the distal part of the duct as in our case that we reported, the injected contrast medium could push the stone proximally, hence making its removal difficult [13].

CT scan and magnetic resonance imaging (MRI), although more complex and expensive than sialography, are currently emerging as the diagnostic tools of choice due to their advantages of accuracy and lower invasiveness. CT scan enables the detection of recently calcified salivary stones, which conventional radiography cannot achieve, although thick radiological slices could occult the stones. However, CT scan is neither capable of localizing the stone precisely within the duct, nor of visualizing the ducts and their anomalies [19]. On the other hand, MRI allows consistent and accurate visualization of salivary stones and the duct system. However, MRI has several disadvantages including distortion artifacts due to dental amalgam, equipment costs and a longer reconstruction duration [20]. Sialendoscopy is a new procedure that can be used either as a diagnostic means by visualizing intraductal stenosis, obstruction and inflammatory changes, or as a treatment option for pathologic conditions [21].

Therapeutic strategy depends on the size and location of the calculi. Conservative treatment, rather than surgical removal, is accepted as the 
first choice in managing small calculi located in the distal section of the duct, expecting their spontaneous expulsion [13].

Hydration should be administered to patients with infrared heating and massage of the gland. Natural sialogogues such as a slice of lemon or medications such as pilocarpine are beneficial as they stimulate the production of saliva and subsequent flushing of calculi. Antibiotics should be initiated whenever infection is suspected to be combined ${ }^{16}$.

Therefore, a surgical approach should be considered if the stone is medium or large in size and the failure of medical treatments is anticipated, leaving only salivary colic.

The treatment of choice, for parotid stones not responding to conservative treatment, is extracorporeal shock-wave lithotripsy under sonographic control. Moreover, it does not require anesthesia, sedation or analgesia. This method is re- ported to be effective, with patient's stone-free in $50-60 \%$ and symptom-free in $80-90 \%$. Although lithotripsy is a useful technique, there is the potential risk of parenchymal damage and fibrosis of the gland [22].

Some authors advocate treatment of sialolithiasis by means of intraductal instillation of penicillin or saline. According to these authors, this method is more effective than systemically administered drugs due to low recurrence rate and many other advantages [1]. When medical therapy is ineffective the next alternative is surgical removal of the calculus or even of the whole gland. One of the disadvantages is facial nerve damage. Intraoral surgery is more effective than extraoral technique because of no visible scar [1].

Parotidectomy should be considered as the last treatment option, in patients with multiple stones ( $>3 \mathrm{~mm}$ stones) in the same gland, recurrent episodes of sialadenitis and after failure of minimal invasive techniques and shockwave lith-otripsy [23].

The literature review highlights the importance of careful history taking and intraoral examination. This should inspire clinicians to assess sialolithiasis with a more comprehensive approach in clinical practice.

This review describes our clinical experience in 8 cases in extraoral approach for removing the intraparotid large sialolith. The patient described in our case was 65 years old and is therefore one of the rare clinical cases of parotid gland sialolithiasis. Presumably, the etiologic factor of calculi in our case appears to be bacterial infection, which triggered an increase in salivary $\mathrm{P}^{\mathrm{H}}$ and an increase of organic matter as well as tissue injury and supersaturation of saliva. But the exact cause remains undetermined, since the patient's laboratory test results were unremarkable as well as his personal history except for the previous infection of parotid gland just 2 weeks before, and it seems not possible for the stone of $4 \mathrm{~mm}$ in size to be formed in such a short time.

The parotid stone in our case couldn't be easily identified by bimanual palpation. That is why we used computerized tomography for differential diagnosis, and only with $\mathrm{CT}$ scan it was possible to identify parotid gland stone. As the stone in our case were near the start point of duct and had $4 \mathrm{~mm}$ size, we decided to do surgical approach without trying conservative treatment.

\section{Conclusion}

Parotid sialolithiasis are less frequent than that of submandibular sialolithiasis, generally unilateral and predominantly affects salivary duct than gland. The patients in our cases had originally been diagnosed with parotid gland sialolithiasis with CT scan and treated with extraoral surgical approach. Extraoral approach for removing the intraparotid large sialolith has been demonstrated to be a reliable technique with good long-term results.

\section{References}

1. Torres-Lagares D, Barranco-Piedra S, Serrera-Figallo MA, Hita-Iglesias P, MartínezSahuquillo-Márquez A, et al. (2006) Parotid sialolithiasis in Stensen's duct. Med Oral Patol Oral Cir Bucal 11: E80-E84. [Crossref]

2. Iqbal A, Gupta AK, Natu SS, Gupta AK (2012) Unusually large sialolith of Wharton's duct. Ann Maxillofac Surg 2: 70-73. [Crossref]

3. Graziani F, Vano M, Cei S, Tartaro G, Mario G (2006) Unusual asymptomatic gian sialolith of submandibular gland. A clinical report. J Craniofacial Surg 17: 549. [Crossref]

4. Wiliams MF (1999) Sialolithiasis. Otolaryngol Clin NA 32: 819-834. [Crossref]

5. Ship JA (2008) Diagnosing, managing, and preventing salivary gland disorders. Oral Dis 8: 77. [Crossref]

6. Eigner TL, Jastak JT, Bennett WM (1986) Achieving oral health in patients with renal failure and renal transplants. J Am Dent Assoc 113: 612-616. [Crossref]

7. Sharma RK, al-Khalifa S, Paulose KO, Ahmed N (1994) Parotid duct stone-removal by a dormia basket. J Laryngol Otol 108: 699-701. [Crossref]

8. Boffano P, Gallesio C (2010) Surgical treatment of a giant sialolith of the Wharton duct. J Craniofac. Surg 21: 134-135. [Crossref]

9. Siddiqui SJ (2002) Sialolithiasis an unusually large submandibular salivary stone. $B r$ Dent J 193: 89-91. [Crossref]

10. Rabinov JD (2000) Imaging of salivary gland pathology. Radiol Clin North Am 38 1047-1057. [Crossref]

11. Lustmann J, Regev E, Melamed Y (1990) Sialolithiasis. A survey on 245 patients and a review of the literature. Int J Oral Maxillofac Surg 19: 135-138. [Crossref]

12. Zenk J, Constantinidis J, Kydles S, Hornung J, Iro H (1999) Clinical and diagnostic findings of sialolithiasis. HNO 47: 963-969. [Crossref]

13. Williams MF (1999) Sialolithiasis. Otolaryngol Clin North Am 32: 819-834. [Crossref]

14. Bodner L (1999) Parotid sialolithiasis. J Laryngol Otol 113: 266-267. [Crossref]

15. Pollack CV, Severance HW (1990) Sialolithiasis: case studies and review. J Emerg Med 8: 561-565. [Crossref]

16. Leung AK, Choi MC, Wagner GA (1999) Multiple sialoliths and a sialolith of unusual size in the submandibular duct: a case report. Oral Surg Oral Med Oral Pathol Oral Radiol Endod 87: 331-333. [Crossref]

17. Gadodia A, Bhalla AS, Sharma R, Thakar A, Parshad R (2011) Bilateral parotid swelling: a radiological review. Dentomaxillofac Radiol 40: 403-414. [Crossref]

18. Avrahami E, Englender M, Chen E, Shabtay D, Katz R, et al. (1996) CT of submandibular gland sialolithiasis. Neuroradiology 38: 287-290. [Crossref]

19. Becker M, Marchal F, Becker CD, Dulguerov P, Georgakopoulos G, et al. (2000) Sialolithiasis and salivary ductal stenosis: diagnostic accuracy of MR sialography with a three-dimensional extended-phase conjugate-symmetry rapid spin-echo sequence. Radiology 217: 347-358. [Crossref]

20. Kim JW, Kim DH, Kim KT, Kim TY, Ko KJ, et al. (2005) Sialendoscopy: endoscopic diagnosis and treatment of the salivary gland disease. Korean J Otolaryngol-Head Neck Surg 48: 373-379.

21. Konstantinidis I, Paschaloudi S, Triaridis S, Fyrmpas G, Sechlidis S, et al. (2007) Bilateral multiple sialolithiasis of the parotid gland in a patient with Sjögren's syndrome. Acta Otorhinolaryn gol Ital 27: 41-44. [Crossref]

22. Zenk J, Werner G, Hosemann M D, Iro H (1998) Diameters of the main excretory ducts of the adult human submandibular and parotid gland - a histological study. Oral Surg Oral Med Oral Pathol Oral Radiol Endod 85: 576-580. [Crossref]

23. Iro H, Schneider HT, Fodra C, Waitz G, Nitsche N, et al. (1992) Shockwave lithotripsy of salivary duct stones. Lancet 339: 1333-1336. [Crossref]

Copyright: (C2020 Papikyn A. This is an open-access article distributed under the terms of the Creative Commons Attribution License, which permits unrestricted use, distribution, and reproduction in any medium, provided the original author and source are credited. 